\title{
Reduced Occipital and Prefrontal Brain Volumes in Dysbindin-Associated Schizophrenia
}

\author{
Gary Donohoe*, ${ }^{*, 2}$, Thomas Frodl 1,2,3, Derek Morris', Ilaria Spoletini ${ }^{4}$, Dara M Cannon ${ }^{5}$, Andrea Cherubini ${ }^{4}$, \\ Carlo Caltagirone $^{4,6}$, Paola Bossù ${ }^{4}$, Colm McDonald ${ }^{5}$, Michael Gill ${ }^{1,2}$, Aiden P Corvin ${ }^{1,2}$ and \\ Gianfranco Spalletta 4 \\ 'Department of Psychiatry, Neuropsychiatric Genetics Research Group, Trinity College Dublin, Dublin, Ireland; ${ }^{2}$ Trinity College Institute for \\ Neuroscience, Trinity College Dublin, Dublin, Ireland; ${ }^{3}$ Department of Psychiatry, Clinical Neuroimaging Research Group, Trinity College Dublin, \\ Dublin, Ireland; ${ }^{4}$ Department of Clinical and Behavioural Neurology, IRCCS Santa Lucia Foundation, Rome, Italy; ${ }^{5}$ Department of Psychiatry, \\ Clinical Neuroimaging Laboratory, National University of Ireland Galway, Galway, Ireland; ' ${ }^{6}$ epartment of Neuroscience, Tor Vergata University, \\ Rome, Italy
}

\begin{abstract}
A three-marker C-A-T dysbindin haplotype identified by Williams et al (PMID: |506689|) is associated with increased risk for schizophrenia, decreased mRNA expression, poorer cognitive performance, and early sensory processing deficits. We investigated whether this same dysbindin risk haplotype was also associated with structural variation in the gray matter volume (GMV). Using voxelbased morphometry, whole-volume analysis revealed significantly reduced GMVs in both the right dorsolateral prefrontal and left occipital cortex, corresponding to the behavioral findings of impaired spatial working memory and EEG findings of impaired visual processing already reported. These data provide important evidence of the influence of dysbindin risk variants on brain structure, and suggest a possible mechanism by which disease risk is being increased.

Neuropsychopharmacology (2010) 35, 368-373; doi: I0.1038/npp.2009. I40; published online 30 September 2009
\end{abstract}

Keywords: schizophrenia; MRl; voxel-based morphometry; dysbindin

\section{INTRODUCTION}

The human dystrobrevin-binding protein 1 (dysbindin) gene located on chromosome 6p22.3 has been associated with schizophrenia risk in multiple independent samples (Riley and Kendler, 2006; Sun et al, 2008). Mutations at dysbindin are recent in evolutionary terms and vary greatly between populations (Guo et al, 2009); reported associations have been heterogeneous, including a six-marker haplotype (Funke et al, 2004) and a three-marker haplotype reported by Williams et al (2004). Investigation of the C-AT-risk haplotype reported by Williams et al was associated with reduced dysbindin mRNA expression in human postmortem analysis of homogenized brain tissue (Bray et al, 2005). The same risk haplotype has also been associated with phenotypic alternations, such as neuropsychological impairments in spatial working memory (Donohoe et al, 2007) and electrophysiological impairments in early visual processing (Donohoe et al, 2008). These findings are complimented by studies of other risk variants and haplotypes at this locus associated with both reduced expression, particularly in the prefrontal and hippocampal

*Correspondence: Dr G Donohoe, Department of Psychiatry, Trinity Health Centre, St James's Hospital, Dublin 8, Ireland, Tel: + 353 I 896 2467, Fax: + 353 | 896 2467, E-mail: donoghug@tcd.ie

Received 19 May 2009; revised 13 July 2009; accepted 29 July 2009 cortex (Talbot et al, 2004; Weickert et al, 2004) and variation in neuropsychological function in both patients and healthy controls (Burdick et al, 2006; Zinkstok et al, 2007; Luciano et al, 2009).

The aim of this study was to determine whether the C-AT-risk haplotype is also associated with structural changes in the gray matter volume (GMV) in patients with schizophrenia. We hypothesized, given the evidence that dysbindin is associated with both general and specific cognitive deficits, that risk carriers would show significantly reduced GMV by comparison with clinically similar patients who were non-risk carriers.

\section{MATERIALS AND METHODS}

\section{Participants}

Participants were drawn from a series of 70 patients with imaging and genetic information and were included on the basis of whether their dysbindin-risk haplotype could be determined (see haplotype analysis below). This resulted in 38 outpatients ( 22 male, mean age 43.3 years, $S D=13.1$ ), with a DSM-IV diagnosis of schizophrenia being included in the analysis. The group was comprised of 18 risk haplotype carriers and 20 non-risk haplotype carriers. All patients were recruited consecutively from two outpatient clinics in central Italy and gave written informed consent to 
participate following local ethics committee guidelines. Exclusion criteria were (i) other axis I or II psychiatric disease except schizophrenia, (ii) a history of traumatic brain injury with loss of consciousness, (iii) a history of epilepsy or seizures, or relevant medical illness (for example, cerebrovascular disease), and (iv) history of substance abuse in the preceding 6 months. All participants were Caucasian and were of Italian ancestry.

\section{Clinical Assessment}

Diagnosis was confirmed using a structured clinical interview (SCID). Clinical history and symptom severity was informed by consultation with patients, their relatives, treating physicians, and a review of the clinical chart. Positive and negative symptoms and general psychopathology were measured using the Positive and Negative Syndrome Scale (PANSS).

Nineteen of the twenty risk haplotype carriers (mean age $39.61, \mathrm{SD}=9.9)$ and sixteen of the eighteen non-risk haplotype carriers (mean age $=40.9, \quad \mathrm{SD}=13.5$ ) were right-handed as assessed by the Edinburgh Handedness Inventory (Oldfield, 1971). All subjects reported normal or corrected-to-normal vision. There were fewer men in the risk carrier group (45\%) vs the non-risk group (69\%), although this difference was not statistically significant $\left(\chi^{2}=2.54, p=0.11\right)$. Age at onset did not differ between groups (mean years for carriers was $25.3(\mathrm{SD}=8.9)$ and mean years for noncarriers was $24.4(\mathrm{SD}=7.1))$; duration of illness was similarly nonsignificant between groups $(t=0.657, \mathrm{df}=36, p=0.516)$. When compared on the PANSS, no difference in symptom severity was observed between groups (mean positive symptoms scale score for carriers was 23.1 $(\mathrm{SD}=7.5)$ vs $24.1 \quad(\mathrm{SD}=9.0)$ for noncarriers, $t(36)=0.986, p \geqslant 0.05$; mean negative symptoms scale scores for carriers was $24.1 \quad(\mathrm{SD}=9.0)$ vs 26.4 $(\mathrm{SD}=8.7)$ for noncarriers, $t(36)=0.783, p \geqslant 0.05)$. All patients were on psychotropic medication at the time of testing. No differences were observed in medication dosage based on chlorpromazine equivalents (risk carriers $512.2 \mathrm{mg}, \mathrm{SD}=399.6$; non-risk carriers $422.3, \mathrm{SD}=223.0$, $\mathrm{F}=0.18, p=0.68)$. The lack of difference between carriers and noncarriers on each of the above variables - age, gender, duration of illness, symptom severity scores, and medication prescribed - accorded with the lack of differences observed in our total genetic sample for these variables ( $n=372$; data available on request).

\section{Neuropsychological Assessment}

Although not a primary focus of this study, a number of indices of neuropsychological performance were also available for patients who participated. Global cognitive status was assessed using the Mini-Mental State Examination (MMSE; Folstein et al, 1983). Processing speed was measured using the Trail-Making Test, parts A and B, and cognitive flexibility was measured using the Wisconsin Card Sorting Test (using both category errors and errors of perseveration). All assessments were carried out by master's degree level psychologists.

\section{Genetic Analysis}

The dysbindin 'risk' haplotype identified by Williams et al (2004) is derived from alleles $C-A-T$ at single nucleotide polymorphisms (SNPs) rs2619539, rs3213207, and rs2619538. All SNPs were genotyped using Custom Taqman SNP Genotyping Assays from Applied Biosystems on an ABI Prism 7900HT Sequence Detection System. Duplicate genotyping in a subset of the total sample from which this MRI sample was drawn (192 of 1200 cases and controls) indicated a genotyping error level of $<1 \%$. Carriers and noncarriers of the haplotype (estimated frequency of 0.21 in cases in the overall sample) were identified using the Bayesian approach to reconstructing haplotypes implemented in the program PHASE (v2.0.2; Stephens and Donnelly, 2003); only cases in which both haplotypes could be identified with $>95 \%$ certainty were included $(\sim 55 \%$ of the total patient imaging sample). None of our risk group were carriers of two copies of the C-A-T-risk haplotype (this was to be expected as in our total genetic studies that only $5 \%$ of the sample carried two copies); therefore, all analysis was carried out as a direct comparison between carriers of one copy of the risk haplotype and noncarriers.

\section{Image Acquisition and Preprocessing}

The magnetic resonance images were acquired at the IRCCS Fondazione Santa Lucia in Rome, Italy, using a $1.5 \mathrm{~T}$ wholebody Siemens Vision Magnetom scanner. T2 and fluidattenuated inversion recovery (FLAIR) sequences were acquired to screen for brain pathology. For voxel-based morphometry analyses, a T1-weighted 3D data set was acquired using a magnetization-prepared rapid gradientecho sequence $\left(\mathrm{TR}=11.4 \mathrm{~ms}, \mathrm{TE}=4.4 \mathrm{~ms}\right.$, flip angle $15^{\circ}$, slice thickness $=1 \mathrm{~mm}$, no interslice gap, voxel si$\mathrm{ze}=1 \times 1 \times 1 \mathrm{~mm})$. Data preprocessing were performed using SPM5 (Wellcome Department of Cognitive Neurology, London, UK) running under MATLAB 6.5 (The MathWorks, Natick, MA, USA).

This study used the VBM5 toolbox, which utilizes and extends the new unified segmentation approach implemented in SPM5 (Ashburner and Friston, 2005). Unified segmentation provides a generative model of VBM preprocessing that integrates tissue classification, image registration, and magnetic resonance imaging in homogeneous bias correction. Thus, the model avoids the 'circularity problem' of the optimized VBM procedure, as the initial image registration does not require an initial tissue segmentation and vice versa (Good et al, 2001). The VBM5 toolbox extends the unified segmentation model, as it increases the quality of segmentation by applying a Hidden Markov Field model on the segmented tissue maps (Bach Cuadra et al, 2005). The Hidden Markov Field algorithm provides spatial constraints based on neighboring voxel intensities within a 3_3_3 voxel cube. It removes isolated voxels, which are unlikely to be a member of a certain tissue class and also closes holes in a cluster of connected voxels of a certain class, resulting in a higher signal-to-noise ratio of the final tissue probability maps. The VBM5 toolbox offers the possibility to write the estimated tissue probability maps without making use of the respective ICBM tissue priors (International 
Consortium for Brain Mapping) from SPM5. This study used this option, as it improved the delineation of the subcortical structures and the sulci in the final tissue maps. The final tissue maps of the gray matter (GM), white matter, and cerebrospinal fluid were modulated with the deformation fields obtained by normalization to standard space to analyze volume differences between study populations. Finally, the modulated GM partitions were smoothed with a $10 \mathrm{~mm}$. FWHM Gaussian kernel and used for statistical analysis.

An analysis of covariance was designed to investigate focal GM volume (GMV) differences between the patients who were carriers of the dysbindin-risk haplotype and patients who were noncarriers. Age and gender were entered as covariates of no interest in the statistical design. First, GMV differences (increases/decreases) were assessed at the whole-brain level using $\mathrm{T}$ contrasts $(p<0.001$ voxel level) and cluster-level family-wise error (FWE) correction ( $p<0.05$, corrected). Cluster size was set at $k \geqslant 300$ voxels and named using WFU Pickatlas.

\section{RESULTS}

On cognitive measures, patients who were risk-haplotype carriers performed below noncarriers on all measures. However, these differences were significant only for the MMSE (risk carriers mean score of 23.2 vs 28.3 for noncarriers; $U=33 ; p=0.04$ ) and not for results on the WCST (categories, perseverative errors) or Trail-Making Task (trail A: $t=-1.10, p=0.28$; trail B: $t=-0.52$, $p=0.61)$.

\section{Whole-Brain Analysis}

On the basis of ANCOVA analysis of GMV differences (in which age and gender were entered as covariates), two clusters were identified in which carriers showed significantly lower GMV (FWE corrected). These were (i) the right dorsolateral prefrontal cortex (DLPFC), with maximal differences located in the right inferior frontal trigonum, as well as in the right middle frontal gyrus, and (ii) the left calcarine cortex with extension to the left superior occipital cortex (anatomical local maxima were identified by means of automated anatomical labeling (Tzourio-Mazoyer et al, 2002)). There were no areas in which risk carriers were observed to have significantly higher GMV than noncarriers (Table 1) (Figure 1).

Table I Between-Group Difference in GMV Between Carriers and Noncarrier Patients of the Dysbindin-Risk Haplotype $(p<0.05$, FWE Cluster Corrected), Maxima MNI Coordinates $(x, y, z)$, Hemisphere ( $L=$ left, $R=$ right), $K$ (Number of Voxels Within Clusters)

\begin{tabular}{lcccc}
\hline Regions & $\boldsymbol{K}$ & $\boldsymbol{T}$ & $\boldsymbol{p}$ corrected (FWE) & $\boldsymbol{x}, \mathbf{y}, \mathbf{z}$ \\
\hline Right frontal inferior trigonum & 802 & 4.25 & $<0.001$ & $43,38,23$ \\
Left calcarine cortex & 390 & 4.12 & 0.003 & $-9,-92,6$ \\
\hline
\end{tabular}

Coordinates of peak significant voxels were assigned to anatomical regions by means of automated anatomical labeling (Tzourio-Mazoyer et al, 2002).

\section{DISCUSSION}

Dysbindin has been associated with both increased schizophrenia risk and variance in cognitive performance. In addition to being associated with reduced mRNA expression, the particular risk haplotype investigated in this study has been previously been associated with deficits in both early visual processing and spatial working memory. Given the correlation between poorer general cognitive performance and smaller GMV (Posthuma et al, 2003; Thompson et al, 2000; Frangou et al, 2004), we hypothesized that dysbindin-risk haplotype carriers would also show smaller GMVs than clinically similar patients who were noncarriers. Supporting this hypothesis, risk carriers were observed to have significantly smaller GMVs in both the right dorsolateral and left occipital cortical regions. Given the often conflicting reports in the risk variants identified at the dysbindin locus, this observation that a haplotype already associated with increased risk, altered mRNA expression, and reduced cognitive performance and electrophysiological response, is also associated with reduced GMV provides additional support that this particular haplotype tags important functionality at the dysbindin locus. Our study focused solely on the risk haplotype that we have previously studied in order to reduce the multiple testing burden associated with testing additional risk variants. Future studies, which compare the relative impact of the various risk variants within the same study design, are likely to be instructive in this regard, as alternative risk variants and haplotypes may implicate additional brain regions.

The fact that the reduced GMV in the right DLPFC and left occipital cortex observed in risk-haplotype carriers in this study are precisely the cortical regions implicated by our earlier associations with spatial working memory and early visual processing is striking, particularly as the present sample includes none of the Irish cases who participated in the earlier studies. A review of 17 twin studies have shown that genetic effects varied regionally within the brain, with highest heritability for the frontal lobe volumes (90-95\%), and moderate estimates in the occipital cortex areas (Peper et al, 2007). Whether owing to the relative heritability of brain volume across different brain regions or for other reasons, the influence of dysbindin appears particularly visible in these regions. This is not to suggest, however, that dysbindin's influence is focal to these regions. Dysbindin is ubiquitously expressed throughout the brain (Allen brain atlas, 2009), and evidence of altered expression associated with other risk variants in other brain regions has already been documented (Weickert et al, 2004), suggesting that dysbindin's influence on brain structure and function is unlikely to be regionally specific. Other schizophrenia-risk variants have already been associated with volumetric differences in the multiple brain regions; for example, NRG1-risk variants have variously been associated with differences in lateral ventricle volume, hippocampal volume, white matter integrity, and regional frontal and temporal activation (McIntosh et al, 2008; Hall et al, 2006).

Given that replication of results is a major issue for psychiatric genetics, especially given the evidence of heterogeneity of risk variants identified, it is interesting to note that a recent study by Narr et al (2009) also reports 
a OCCIPITAL CORTEX

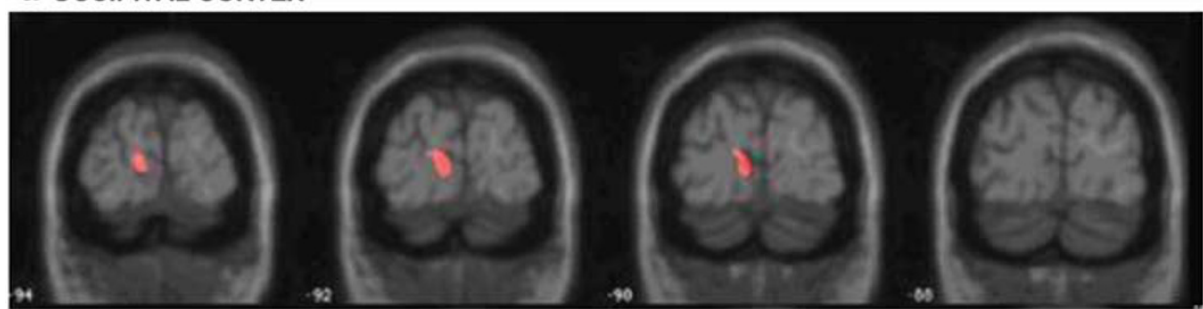

b PREFRONTAL CORTEX

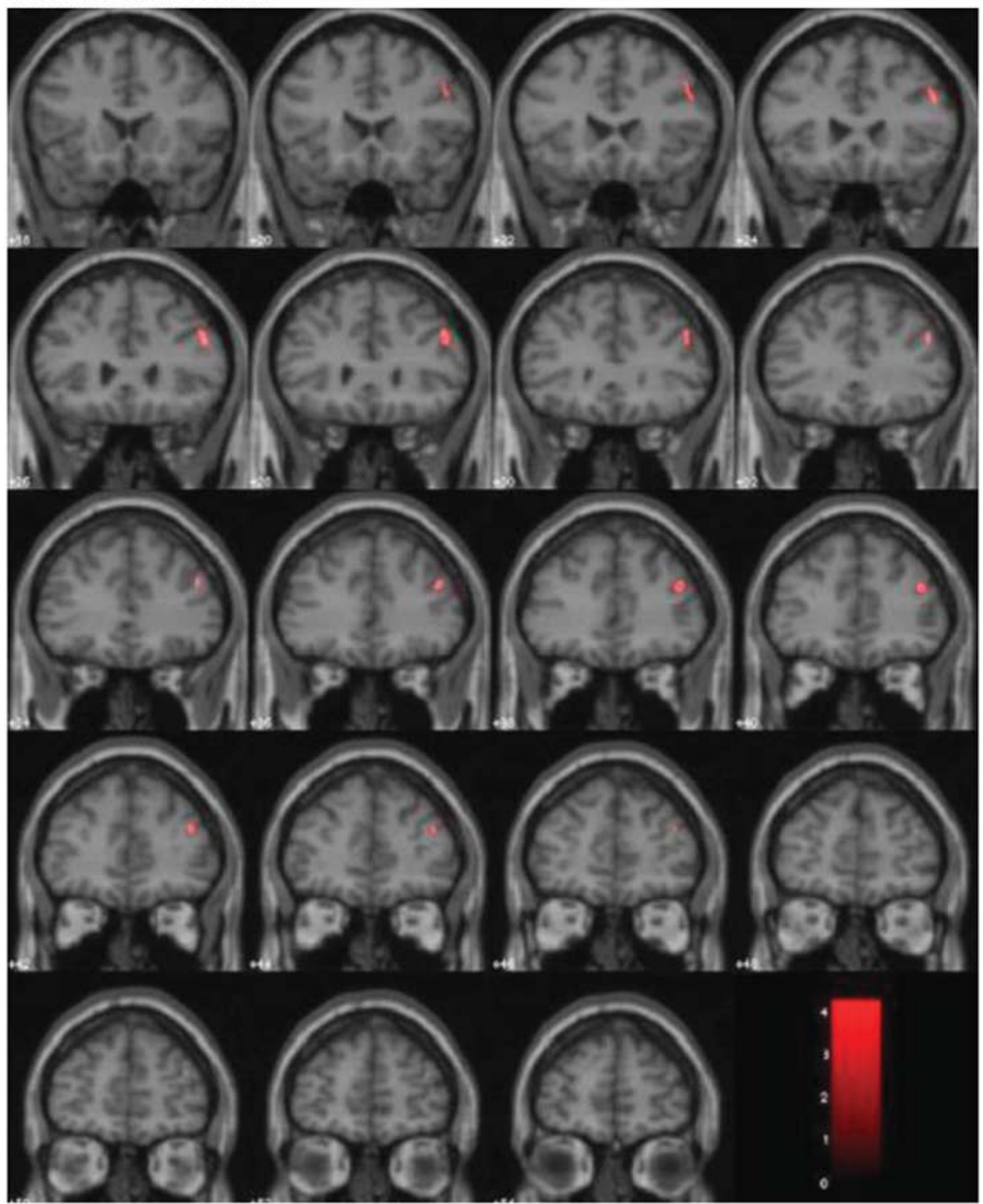

Figure I Differences between patient carriers and non-carriers of the dysbindin-risk haplotype. Risk carriers show significantly smaller gray matter volumes in (a) left occipital cortex and (b) right dorsolateral prefrontal cortex (FWE corrected).

evidence of dysbindin's influence on brain function. In this study a risk variant at the SNP rs1018381, which was independent of those SNPs contributing to the risk haplotype reported here, was associated with both reduced brain volume and variation in cortical thickness in patients (a measure highly correlated with VBM-derived measure of the GM). In terms of effect size, calculation of differences in brain volume associated with dysbindin genotype (based on the reported F-statistics) resulted in an effect size $d=0.60$, usually considered a 'medium' effect size as described by Cohen (1992). In our own study, effect sizes associated with the T-values observed for prefrontal and occipital differences were 1.36 and 1.34, respectively, both large effects based on Cohen's criteria, comparable with the earlier effect sizes observed in our previous EEG study of early visual processing associated with dysbindin (Donohoe et al 2008). Given the likelihood that many genetic variants are likely to influence gene function, this suggests that 
dysbindin, in addition to other schizophrenia-risk variants such as NRG1 for which similar effects sizes have been reported (Mata et al, 2009), is among the more important genetic determinants of structural alterations in brain volume in schizophrenia.

In addition to noting cortical thinning in the occipital pole, Narr et al also report evidence of cortical thinning in patients in other areas, including superior and middle temporal gyri, and supragenual and paracingulate cortices. This again emphasizes the apparently general rather than focal influence of dysbindin on brain structure and the likelihood that alternative dysbindin-risk variants may have greater structural influence on the cortical regions other than the region reported here for the C-A-T-risk haplotype. Furthermore, in specifying the selective effects of dysbindin on brain structure in patients $v s$ controls, cortical thinning was only noted for patients and not healthy controls in the Narr et al study, whereas reduced brain volume was noted in both groups. The absence of a healthy comparison group in this study is a drawback that precludes discussion of the effects of the risk haplotype on brain volume in healthy controls and investigation of the risk variants reported here in relation to brain structure alterations in healthy control is warranted.

The precise mechanism by which dysbindin contributes to variance in brain structure and function remains unclear. Dysbindin has previously been implicated in both glutamate function through presynaptic vesicular trafficking (Numakawa et al, 2004), and dopaminergic function through DRD2 availability (Iizuka et al, 2007). Dysbindin is also reported to have a wide range of protein-protein interacting partners, which have been grouped in terms of (i) cell morphology and cellular development, and (ii) cell-cell signaling and developmental disorders. Whether the influence on prefrontal volume reported here represents a specific effect of dysbindin, or is a consequence of its interaction with a protein partner, is yet to be determined. In conclusion, the evidence of dysbindin's influence on brain structure in this study provides important confirmation of its role in the brain development.

\section{ACKNOWLEDGEMENTS}

We sincerely thank those patients who participated in this study and the staff who facilitated their involvement. Dr Donohoe is generously supported by a NARSAD young investigator grant and a Science Foundation Ireland Research Frontiers award. Professor Frodl is supported by a Science Foundation Ireland Stokes grant.

\section{DISCLOSURE}

The authors declare no conflict of interest.

\section{REFERENCES}

Allen brain atlas (2009). Allen Institute for Brain Science. http:// www.brain-map.org Accessed on 15 June 2009.

Ashburner J, Friston KJ (2005). Unified segmentation. Neuroimage 26: $839-851$.

Bach Cuadra M, Cammoun L, Butz T, Cuisenaire O, Thiran JP (2005). Comparison and validation of tissue modelization and statistical classification methods in T1-weighted MR brain images. IEEE Trans Med Imaging 24: 1548-1565.

Bray NJ, Preece A, Williams NM, Moskvina V, Buckland PR, Owen MJ et al (2005). Haplotypes at the dystrobrevin binding protein 1 (DTNBP1) gene locus mediate risk for schizophrenia through reduced DTNBP1 expression. Hum Mol Genet 14: 1947-1954.

Burdick KE, Lencz T, Funke B, Finn CT, Szeszko PR, Kane JM et al (2006). Genetic variation in DTNBP1 influences general cognitive ability. Hum Mol Genet 15: 1563-1568.

Cohen J (1992). 'A power primer'. Psychol Bull 112: 155-159.

Donohoe G, Morris DW, Clarke S, McGhee KA, Schwaiger S, Nangle JM et al (2007). Variance in neurocognitive performance is associated with dysbindin-1 in schizophrenia: a preliminary study. Neuropsychologia 45: 454-458.

Donohoe G, Morris DW, De Sanctis P, Magno E, Montesi JL, Garavan HP et al (2008). Early visual processing deficits in dysbindin-associated schizophrenia. Biol Psychiatry 63: 484-489.

Folstein MF, Robins LN, Helzer JE (1983). The mini-mental state examination. Arch Gen Psychiatry 40: 812.

Funke B, Finn CT, Plocik AM, Lake S, DeRosse P, Kane JM et al (2004). Association of the DTNBP1 locus with schizophrenia in a U.S. population. Am J Hum Genet 75: 891-898.

Frangou S, Chitins X, Williams SC (2004). Mapping IQ and gray matter density in healthy young people. Neuroimage 23: 800-805.

Good CD, Johnsrude IS, Ashburner J, Henson RN, Friston KJ, Frackowiak RS (2001). A voxel-based morphometric study of ageing in 465 normal adult human brains. Neuroimage 14: 21-36.

Guo AY, Sun J, Riley BP, Thiselton DL, Kendler KS, Zhao Z (2009). The dystrobrevin-binding protein 1 gene: features and networks. Mol Psychiatry 14: 18-29.

Hall J, Whalley HC, Job DE, Baig BJ, McIntosh AM, Evans KL et al (2006). A neuregulin 1 variant associated with abnormal cortical function and psychotic symptoms. Nat Neurosci 9: 1477-1478.

Iizuka Y, Sei Y, Weinberger DR, Straub RE (2007). Evidence that the BLOC-1 protein dysbindin modulates dopamine D2 receptor internalization and signalling but not D1 internalization. J Neurosci 27: 12390-12395.

Luciano M, Miyajima F, Lind PA, Bates TC, Horan M, Harris SE (2009). Variation in the dysbindin gene and normal cognitive function in three independent population samples. Genes Brain Behav 8: 218-227.

McIntosh AM, Moorhead TW, Job D, Lymer GK, Muñoz Maniega S, McKirdy J (2008). The effects of a neuregulin 1 variant on white matter density and integrity. Mol Psychiatry 13: 1054-1059.

Narr KL, Szeszko PR, Lencz T, Woods RP, Hamilton LS, Phillips $\mathrm{O}$ et al (2009). DTNBP1 is associated with imaging phenotypes in schizophrenia. Hum Brain Mapp; e-pub ahead of print 15 May 2009.

Numakawa T, Yagasaki Y, Ishimoto T, Okada T, Suzuki T, Iwata N et al (2004). Evidence of novel neuronal functions of dysbindin, a susceptibility gene for schizophrenia. Hum Mol Genet 13: 2699-2708.

Oldfield RC (1971). The assessment of handedness: the Edinburgh inventory. Neuropsychologia 9: 97-113.

Peper JS, Brouwer RM, Boomsma DI, Kahn RS, Hulshoff Pol HE (2007). Genetic influences on human brain structure: a review of brain imaging studies in twins. Hum Brain Mapp 28: 464-473.

Posthuma D, Baaré WF, Hulshoff Pol HE, Kahn RS, Boomsma DI, De Geus EJ (2003). Genetic correlations between brain volumes and the WAIS-III dimensions of verbal comprehension, working memory, perceptual organization, and processing speed. Twin Res 6: 131-139.

Riley B, Kendler KS (2006). Molecular genetic studies of schizophrenia. Eur J Hum Genet 14: 669-680. 
Stephens M, Donnelly P (2003). A comparison of bayesian methods for haplotype reconstruction from population genotype data. Am J Hum Genet 73: 1162-1169.

Sun J, Kuo PH, Riley BP, Kendler KS, Zhao Z (2008). Candidate genes for schizophrenia: a survey of association studies and gene ranking. Am J Med Genet B Neuropsychiatr Genet 147B: 1173-1181.

Talbot K, Eidem WL, Tinsley CL, Benson MA, Thompson EW, Smith RJ et al (2004). Dysbindin-1 is reduced in intrinsic, glutamatergic terminals of the hippocampal formation in schizophrenia. J Clin Invest 113: 1353-1363.

Thompson PM, Giedd JN, Woods RP, MacDonald D, Evans AC, Toga AW (2000). Growth patterns in the developing brain detected by using continuum mechanical tensor maps. Nature 404: 190-193.

Tzourio-Mazoyer N, Landeau B, Papathanassiou D, Crivello F, Etard O, Delcroix N et al (2002). Automated anatomical labeling of activations in SPM using a macroscopic anatomical parcellation of the MNI MRI single-subject brain. Neuroimage 15: 273-289.

Weickert CS, Straub RE, McClintock BW, Matsumoto M, Hashimoto R, Hyde TM et al (2004). Human dysbindin (DTNBP1) gene expression in normal brain and in schizophrenic prefrontal cortex and midbrain. Arch Gen Psychiatry 61: 544-555.

Williams NM, Preece A, Morris DW, Spurlock G, Bray NJ, Stephens $\mathrm{M}$ et al (2004). Identification in 2 independent samples of a novel schizophrenia risk haplotype of the dystrobrevin binding protein gene (DTNBP1). Arch Gen Psychiatry 61: 336-344.

Zinkstok JR, de Wilde O, van Amelsvoort TA, Tanck MW, Baas F, Linszen DH (2007). Association between the DTNBP1 gene and intelligence: a case-control study in young patients with schizophrenia and related disorders and unaffected siblings. Behav Brain Funct 3: 19-24. 